\title{
A Mechanism for Evaluating Feedback of E-Commerce Sites
}

\author{
Karen Renaud, Paula Kotzé, and Tobias van Dyk \\ Department of Computer Science and Information Systems, University of South Africa
}

\begin{abstract}
The developers of e-commerce applications have a problem in gauging the knowledge and expertise of end-users. The developer must therefore design the interface to the system so that the use thereof is sufficiently intuitive to require the minimum of background knowledge. Developers need to enhance the usability of their web sites so that incidental users will find the site rewarding and enticing, and encourage them to explore further. This paper will explore the role of feedback as a valuable tool in enhancing the interpretability of e-commerce applications. We discuss firstly how applications may be designed to make use of an enriched model of application feedback, and secondly how developers may evaluate their sites so as to gauge the efficacy of the currently provided feedback. We propose a novel method for analysing the purchasing phase of the e-commerce experience. We then introduce an evaluation method for determining whether a particular site provides adequate feedback or not. Three sites were evaluated using the proposed model, and the results of this evaluation are analysed.
\end{abstract}

\section{INTRODUCTION}

Organisations can hardly afford to ignore the E-Commerce (EC) alternative to traditional marketing [1]. EC users will seldom complain about badly-designed sites and the first inkling of a problem may come only from analysis of usage patterns which will very possibly come too late to enable corrective action to be taken, or damage to be prevented. Such patterns, as derived from logs, are very difficult to interpret [13], as is demonstrated by Rosenstein in his study of server logs [20]. According to Rohn [19], there are two important things an EC site should do in order to be successful:

- attract additional customers, and

- reduce workload for the sales force. 
Keeping current customers might be even more important [9]. EC purveyors can never become complacent because EC frees users from restraints which previously forced them to use sub-standard retail stores $[8,13]$. Many researchers rate ease of use as being of critical importance to the EC process [11]. Bad usability is often blamed for causing the failure of sites $[9,19]$.

In this paper we have chosen to discuss one particular aspect of web-site usability, namely that of feedback. We will advocate the extensive use of feedback to increase the interpretability of systems, thus enhancing the ease of use of these systems. Section 2 will explore the nature of feedback in EC

applications. It is necessary, however, to convert this discussion about the merits of feedback to a methodology for evaluating proposed EC web site pages. Before such a methodology can be provided it is necessary to understand the nature of the EC shopping experience, and this will be discussed in Section 3. Section 4 proposes a feedback evaluation methodology for EC systems. Section 5 discusses the results of an evaluation which was conducted on three large EC sites. Section 6 concludes.

\section{FEEDBACK}

Feedback serves a behavioural purpose in the interaction between users and computers, with the computer fulfilling the same role as a conversational participant [16]. Only by means of feedback can participants in a conversation detect faults in the understanding of what is said [5]. The success of the human-computer 'conversation' will depend on the user being able to gauge the 'knowledge' of the application. Feedback must make the 'knowledge' of the application, based on previous inputs, tangible and accessible in order to fulfil its role adequately in the face of an untutored and unreliable user population.

The conversational model of user interaction, with respect to the current computer usage paradigm of recognition rather than recall [4], leads us to consider users as reacting according to the way they interpret the state of the system. The quality of the feedback provided by the system can assist in enabling an understanding of the state of the system and becomes very important when the system is prone to long response times, a common occurrence in e-commerce systems.

\subsection{Use of Feedback}

It is necessary to consider the purpose of any feedback, and the way a user can be expected to make use of such feedback as is provided. The Oxford English Dictionary (OED) defines feedback as: signifying a response, modifying the behaviour of the user and promoting understanding. The traditional role of feedback in human-computer interaction is often seen exclusively as pertaining to the first use. 
The extension of the feedback concept to include all the features will enable EC sites to give better and adequate feedback to users.

It has been noted by various researchers that a discourse typically has an incremental quality about it [3]. Dix [4] argues that it is difficult for users to manage and visualise this "sense of history" in their interaction with the computer. Often the application's only concession to a user's need for this is the provision of an undo facility. This provides a type of historical function but the user often needs to reverse the state of the system in order to see what happened before.

It is therefore appropriate to consider the need for the portrayal of previous system states so that the user can refer to it in order to understand the present state of the system. Historical functions are routinely provided by web browsers, such as a history of sites visited and bookmarks, but we feel that this history should be more finely grained than that. The user needs to have a history of their interaction within particular web sites. We postulate that good feedback should involve giving the user both immediate and archival feedback. There are several difficulties in providing such feedback, such as the difference in technical expertise between the developers and the users of e-commerce sites, the naïvety and unknown nature of e-commerce end-users, and the distributed nature of e-commerce systems, which makes them prone to outages and unpredictable behaviour.

\subsection{Recommendations}

Developers need to have guidelines to ensure that adequate feedback is provided. This section draws the conclusion that developers need to provide the following:

- immediate feedback: signal a response to each user action, explain unusual occurrences such as delays, display relevant system state clearly.

- archival feedback: provide a historical function which allows users to check on previous actions without changing the state of the system. Always provide the facility for users to check on their progress through any process which has specific stages.

This feedback satisfies the OED definition of the purpose of feedback. Having defined feedback, we now develop a model of the EC shopping process. This will enable us to arrive at an EC-specific set of guidelines for determining feedback quality. The next section provides such a model, and Section 4 uses this model and the findings of this section to derive the required guidelines. 


\section{ANALYSIS OF THE E-COMMERCE PURCHASING EXPERIENCE}

Guttman et al. [7] identify six stages of customer purchasing behaviour: need identification, product brokering, merchant brokering, negotiation, purchase and delivery, and service and evaluation. O'Keefe and McEachern [14] propose a model with only five processes: need recognition, information search, evaluation, purchase, and after- purchase evaluation. Singh et al. [21] break up the EC process into three activities: identifying and finding a vendor, purchasing and tracking. We will examine only one of Singh's processes - namely the one that everyone refers to as the purchase task. This task can be split up into two distinct stages, as shown in Figure 1.

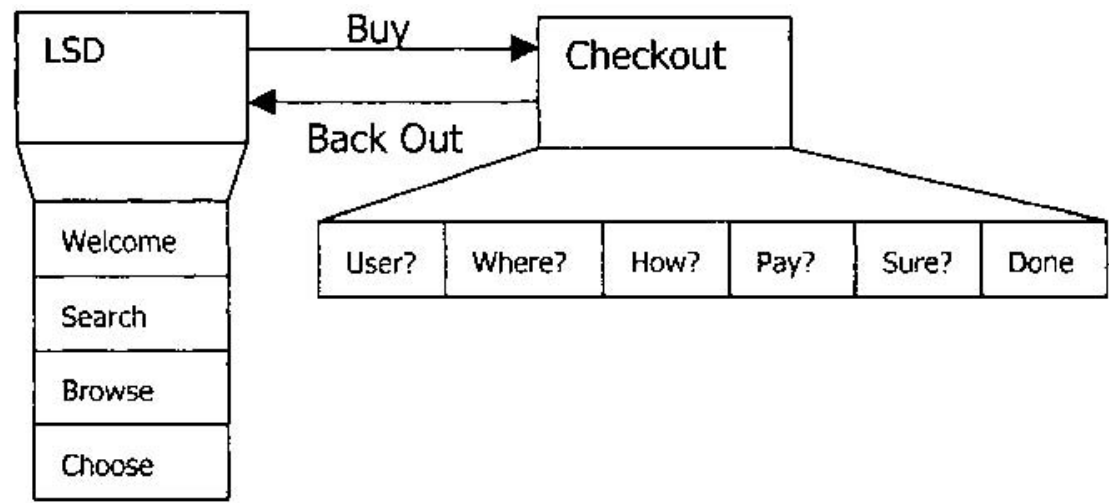

Figure 1. The Two Phases and Ten Stages of the Purchase Task.

1. Look, See and Decide (LSD): This stage will typically be used to look at available products, compare them, and to make a decision about whether or not to purchase products. This may be done one or more times until the consumer has found products which satisfy his or her needs. This phase is intensely user-driven because the user is looking at and assimilating information continuously. It has the following substages which can be traversed iteratively and in varying sequences: Welcome; Search; Browse; and Choose.

2. Checkout: When the users trigger this stage they have made their choice of offered products and decided to make a purchase. They now have to provide certain details, such as their address and credit card details. This stage is system-driven and changes the paradigm of the interaction process from user initiative to system initiative. Feedback is of critical importance during this stage - users who feel that they have lost control can simply leave the site without any embarrassment - unlike a user who is standing at a checkout till in a supermarket. This stage is typically 
composed of at least the following steps, which should be navigated in a serial fashion: User? Where? How? Payment? Sure? and Done. Only one of the previous steps has elicited much research interest ie. Payment [10].

\section{METHOD}

The feedback and information requirements of a user during the LSD stage are very different from those required by the checkout stage. The differences in operation between these phases make it beneficial to develop two different strategies for evaluating web pages since some sites will support one stage far better than the other and one single evaluation process is unlikely to suit both stages.

In deriving an evaluation method it was necessary to combine the findings of various researchers. Ravden and Johnson [17] propose a checklist-based evaluation mechanism which rates interfaces from various perspectives such as visual clarity, consistency, compatibility, explicitness, appropriate functionality, informative feedback, control, error handling and finally, user guidance and support.

We have selected elements from the most relevant of Ravden and Johnson's categories in order to set up one complete feedback evaluation mechanism, for each stage, which will ensure that an EC page provides adequate and complete feedback. The evaluation metrics for the LSD phase are shown in Table 1.

The metrics for the checkout phase are somewhat different, because of the linear and structured nature of the process. The metrics are given in Table 2. The following section will describe how these metrics were applied to three EC sites, and comment about the efficacy of the proposed evaluation mechanism. In order to evaluate EC web pages, a score is given for each of the above questions as follows:

- Never (0) - the feature is never available.

- Sometimes (1) - the feature is seldom there.

- Mostly (2) - the feature is usually there.

- Always (3) - the feature is universally available.

The scores are then determined per stage, and per site, in the form of a percentage where $100 \%$ indicates a site giving a user perfect feedback and sites scoring $0 \%$ might as well give up and close shop. The scores per feature in each stage were calculated by adding up the score for each page making up the stage and awarding a total for each particular feature. The scores for each feature were then totalled to arrive at a percentage per site per purchasing stage.

\section{EVALUATION}

We chose three sites to apply the metrics to. Booksellers like Amazon (www.amazon.ac.uk) are the pioneers in this field and we felt that their site would 
be a good one to evaluate. We therefore chose two other bookseller's sites to compare it to - namely Books Online (www.uk.bol.com) and Kalahari (www.kalahari.net). We purchased various products from each EC site, and evaluated the process. Our final scores for each site, arrived at after discussion and consensus of all the authors, are given in Tables 1 and 2.

\begin{tabular}{|l|l|l|l|l|}
\hline $\begin{array}{l}\text { Evaluation of System Feedback Quality: } \\
\text { LSD Stage }\end{array}$ & $\begin{array}{l}\text { Amazon } \\
\text { Max 9 }\end{array}$ & $\begin{array}{l}\text { Kalahari } \\
\text { Max 9 }\end{array}$ & $\begin{array}{l}\text { BOL } \\
\text { Max 9 }\end{array}$ \\
\hline 1 & Is it clear what a user must do to search for a product? [9, 13] & 6 & 6 & 7 \\
\hline 2 & Does the search engine offer alternatives if a search fails?[10] & 9 & 3 & 0 \\
\hline 3 & Does the system inform the user of the reasons for delays? [12] & 5 & 3 & 3 \\
\hline 4 & Are different types of information clearly separated? [2] & 7 & 5 & 9 \\
\hline 5 & Was information readily available? [9, 15] & 6 & 6 & 6 \\
\hline 6 & Is it clear what needs to be done to select a product? & 9 & 6 & 6 \\
\hline 7 & Can the user undo a product selection? [12] & 9 & 6 & 8 \\
\hline 8 & Is it clear what must be done to make the transition to checkout? & 6 & 6 & 9 \\
\hline 9 & Does the system allow users to check on previous searches? [18] & 0 & 0 & 0 \\
\hline 10 & Is jargon user-centric?[12] & 6 & 6 & 6 \\
\hline 11 & Is there a help facility?[22, 15] & 9 & 9 & 9 \\
\hline & Percentage & $73 \%$ & $57 \%$ & $64 \%$ \\
\hline
\end{tabular}

Table 1: Evaluation Metrics for the LSD Stage

\subsection{Discussion}

One notices from Tables 1 and 2 that some metrics scored markedly well or badly. A low score should wave a red flag at the developer and can be used to indicate a problem area. A high score shows that the developer has one a good job in providing adequate feedback for that particular feature of the site. This section will discuss the low and high scoring feedback features of the three sites. 


\begin{tabular}{|l|l|l|l|l|}
\hline \multicolumn{2}{|l|}{$\begin{array}{l}\text { Evaluation of System Feedback Quality: } \\
\text { Checkout Stage }\end{array}$} & $\begin{array}{l}\text { Amazon } \\
\text { Max 18 }\end{array}$ & $\begin{array}{l}\text { Kalahari } \\
\text { Max 9 }\end{array}$ & $\begin{array}{l}\text { BOL } \\
\text { Max 15 }\end{array}$ \\
\hline 1 & Are Instructions and messages concise and positive? [13] & 12 & 6 & 9 \\
\hline 2 & Are Instructions clear and prompts unambiguous? [13] & 12 & 6 & 12 \\
\hline 3 & Are possible actions clear? & 11 & 5 & 11 \\
\hline 4 & Is It clear what a user must do to take action? [9,13] & 13 & 5 & 13 \\
\hline 5 & Is the required format of user inputs clearly indicated? 15] & 15 & 7 & 10 \\
\hline 6 & Are user actions linked to changes in the interface? & 13 & 6 & 12 \\
\hline 7 & Is there always an appropriate response to user actions? & 12 & 6 & 13 \\
\hline 8 & $\begin{array}{l}\text { Does the system inform the user of the success or failure of } \\
\text { theiractions? }\end{array}$ & 14 & 6 & 11 \\
\hline 9 & Does he system inform the user of the reasons for delays? [11] & 11 & 3 & 5 \\
\hline 10.1 & Do error messages indicate: & & & \\
\hline 10.2 & What errors are? & 12 & 6 & 13 \\
\hline 10.3 & Where errors are? & 6 & 3 & 10 \\
\hline 10.4 & Why they have occurred? & 6 & 1 & 13 \\
\hline 11 & Is It clear what the user has to do to complete the task? & 11 & 5 & 8 \\
\hline 12 & Does the system indicate the current stage? [22] & 17 & 3 & 14 \\
\hline 13 & Was information always readily available? [9,15] & 9 & 6 & 12 \\
\hline 14 & Can the user easily back out of the process? [12] & 10 & 2 & 3 \\
\hline 15 & Is final purchase is confirmed by the user? & 18 & 9 & 15 \\
\hline 16 & Can user check on inputs provided during the process? [18] & 9 & 6 & 3 \\
\hline 17 & is jargon and terminology user-centric? & 12 & 6 & 9 \\
\hline 18 & Is there a help facility? [22,15] & 16 & 9 & 15 \\
\hline & Percentage & $66 \%$ & $59 \%$ & $72 \%$ \\
\hline
\end{tabular}

Table 2: Evaluation Metrics for the Checkout Phase

\subsubsection{Low scores}

Two criteria stand out particularly: the lack of a historical facility and inadequate reasons for long or unexpected delays. None of the evaluated sites allow users to remind themselves of previous search criteria. A user searching for a specific type of book may type in many different search parameters and may easily forget which parameters have been tried before, especially after a period of time has elapsed. It would be helpful to have a drop-down menu which can be activated by the user in order to see previous search criteria.

In the same vein, there is also a need for the user to be reminded, as they progress through the checkout stage, of their previous inputs. Some sites do provide this but it is often not done consistently. 
The other controversial score is the one allocated to the question: Does the system inform the user of the reasons for delays? Most browsers give observable feedback on page-fetch delays and anticipated completion times. However, many sites, including the ones evaluated, seem to rely completely on this facility rather than providing the user with some sort of site-specific indicator of site access (hitrate). A user who is given access to such an indicator will perhaps be more patient when sites take a long time to respond.

A feature which we had considered to be essential and basic to good practice, namely that of indicating the substage throughout the checkout stage, was almost absent in the Kalahari site. The user becomes disoriented because the checkout stage encompasses various substages and they have no way of knowing where they are in the process.

\subsubsection{High Scores}

Some positive features should also be mentioned. The e-mail confirmation sent out by all three sites is a very good feature. The scores allocated to help facilities were high for all the sites - and this is particularly good with respect to site usability. What is good for an EC user is automatically good for the site too.

Another universal feedback feature is the requirement that the user positively confirm their transaction. This eliminates possible errors later when users find that they have made a mistake and not picked it up in time. All evaluated sites offer a final page which displays all choices made for the transaction, allows the user to check these choices, and waits for the user to confirm before processing the order. All sites also send the user an e-mail confirming the order so that the user can still exercise a form of recovery, via e-mail, if he or she wishes to cancel the order.

Amazon always attempts to offer alternatives when a search does not produce any results. Another feature provided by both Amazon and BOL is their usage of a customer-driven rating system. Although this is not feedback in the traditional EC sense it is undeniably valuable to potential customers.

\section{CONCLUSION}

Feedback can be used to assist the user in understanding the functionality and requirements of an EC application and can be effectively harnessed to ensure that users do not simply give up on sites. We have identified two distinct and dissimilar phases during the shopping cycle and have applied stage-specific evaluation metrics to them. This provides an evaluation mechanism which can be used by developers to flag problem feedback areas. 


\section{REFERENCES}

[1]K. Bennet and J. Slonim. The electronic commerce position paper. ACM Computing Surveys, 28(4es):112, 1996.

[2] J. A. Borges, I. Morales, and N. J. Rodriguez. Page Design Guidelines Developed Through Usability Testing. In [8], chapter 11, pages 137 - 152. 1998.

[3]G. Carenini and J. D. Moore. Generating Explanations in Context. In Proceedings of the 1993 International Workshop on Intelligent User Interfaces, Session 6: User Support, pages $175-182,1993$.

[4] A. J. Dix. Closing the loop: modelling action, perception and information. In M. F. C. T. Catarci, S. Levialdi, and G. Santucci, editors, AVI'96 - Advanced Visual Interfaces, pages 20-28. ACM Press, 1991.

[5]F. L. Engel and R. Haakma. Expectations and Feedback in User-System Communication. International Journal of Man-Machine Studies, 39(3):427-452, 1993.

[6]C. Forsythe, E. Grose, and J. Ratner, editors. Human Factors and Web Development. Lawrence Erlbaum, Mahwah, New Jersey, 1998.

[7]R. H. Guttman and A. G. M. ans P Maes. Agent-mediated electronic commerce: a survey. Knowledge Engineering Review, 13 : 147- 159, 1998.

[8]G. Huaubl and V. Trifts. Cosumer decision Making in Online Shopping Environments: The Effects of Interactive Decision Aids. Marketing Science, 19(1):4-21, Winter 2000.

[9]M. G. Helander and H. M. Khalid. Modeling the customer in electronic commerce. Applied Ergonomics, 31:609-619, 2000.

[10] J. Jahng, H. Jain, and K. Ramamurthy. Effective Design of Electronic Commerce Environments: A Proposed Theory of Congruence and an Illustration. IEEE Transactions on Systems, Man and Cybernetics - Part A: Systems, and Humans., 30(4):456-471, July 2000.

[11] G. E. Miles and A. Howes. Framework for understanding human factors in web-based electronic commerce. International Journal of Human Computer Studies, 52(1): 131-163, 2000.

[12] J. Nielsen. useit.com: Jakob nielsen's website. useit.com: usable information technology. http://www.useit.com.

[13] J. Nielsen and D. Norman. Usability on the Web Isn't A Luxury. Information Week, January 14 2000. http://www.informationweek.com/773/web.htm.

[14]R. M. O'Keefe and T. McEachern. Web-based customer decision support systems. Communications of the ACM, 41:71-78, 1998.

[15] P. Paper. Making Online Information Usable. http://www.world.std.com/ uieweb/online.htm.

[16] M. A. Perez-Quinones and J. L. Sibert. Negotiating User-Initiated Cancellation and Interruption Requests. In Proceedings of ACM CHI 96 Conference on Human Factors in Computing Systems, volume 2 of SHORT PAPERS: Models, pages 267-268, 1996.

[17]S. J. Ravden and G. I. Johnson. Evaluating Usability of Human-Computer Interfaces: A Practical Method. John Wiley and Sons, 1989.

[18] K. Renaud and R. Cooper. Feedback in Human-Computer Interaction - Characteristics and Recommendations. South African Computing Journal, (26), 2000.

[19] J. A. Rohn. Creating usable E-commerce sites. STDVIEW: Standard View, the ACM Journal on Standardization, 6, 1998.

[20] M. Rosenstein. What is actually taking place on web sites: e-commerce lessons from web server logs. In Proceedings of the 2nd ACM conference on Electronic commerce, pages 38-43, Minneapolis, MN USA, October 17 - 20, 2000. 
[21] M. Singh, A. K. Jain, and M. P. Singh. E-Commerce over communicators: Challenges and solutions for user interfaces. In Proceedings of the ACM Conference on Electronic Commerce (EC-99), pages 177-186, N.Y., Nov. 3-5 1999. ACM Press.

[22] R. Tilson, J. Dong, S. Martin, and E. Kieke. A Comparison of Two Current E-Commerce Sites. In ACM 16th International Conference on Systems Documentation, Web Navigation, pages 87-92, 1998. 